Korean J. Math. 21 (2013), No. 2, pp. 189-195

http://dx.doi.org/10.11568/kjm.2013.21.2.189

\title{
A STUDY ON THE CARTESIAN CLOSED CATEGORY POSM
}

\author{
IG SUng KIM
}

\begin{abstract}
PosM is a category whose objects are ample spaces and morphisms are possibility mappings. We study some properties of the Category PosM . So we show that Category PosM is a cartesian closed category, and it forms a topos with some condition.
\end{abstract}

\section{Introduction}

Yuan [4] showed that PosM, whose objects are ample spaces and morphisms are possibility mappings, is a category.

In this paper, we study some properties of the Category PosM. In particular, terminal object, equalizer, finite product, pull-back and exponentials exist in the Category PosM. So Category PosM is a cartesian closed category. Also with some condition, it forms a topos.

\section{Preliminaries}

In this section, we state some definitions and properties which will serve as the basic tools for the arguments to prove our results.

Definition 2.1. Let $X$ be a set and $\mathcal{A}$ be a subset of power set $P(X)$ of $X$.

If

Received April 6, 2013. Revised May 19, 2013. Accepted May 25, 2013.

2010 Mathematics Subject Classification: 18B25.

Key words and phrases: category PosM, cartesian closed category, ample space, possibility mapping.

(c) The Kangwon-Kyungki Mathematical Society, 2013.

This is an Open Access article distributed under the terms of the Creative commons Attribution Non-Commercial License (http://creativecommons.org/licenses/by -nc/3.0/) which permits unrestricted non-commercial use, distribution and reproduction in any medium, provided the original work is properly cited. 
(1) $X \in \mathcal{A}$

(2) $A \in \mathcal{A} \Rightarrow A^{c} \in \mathcal{A}$

(3) For any index set $I, A_{i} \in \mathcal{A} \Rightarrow \cup A_{i} \in \mathcal{A}$.

Then $A$ is called an ample field over $X$ and $(X, \mathcal{A})$ is called an ample space.

Definition 2.2. Let $(X, \mathcal{A})$ be an ample space, then $[x]=\bigcap\{A \mid x \in A \in \mathcal{A}\}$

is called an atom of $\mathcal{A}$ containing the element $x \in X$.

Proposition 2.3. Let $(X, \mathcal{A})$ be an ample space, then

(1) $[x] \subseteq A$ or $[x] \cap A=\varnothing$ for any $A \in \mathcal{A}$

(2) $A \in \mathcal{A} \Leftrightarrow A=\cup[x]$

Proof. See [4], [5].

Definition 2.4. Let $(X, \mathcal{A})$ be an ample space. If a mapping $\Pi$ : $\mathcal{A} \rightarrow[0,1]$ satisfies

(1) $\Pi(\emptyset)=0 ; \Pi(X)=1$;

(2) $\Pi\left(\cup A_{i}\right)=\sup \Pi\left(A_{i}\right)$.

Then $\Pi$ is called a possibility measure over $\mathcal{A}$, and $M(x)=\Pi([x])$ is called a possibility distribution of $\Pi$.

Definition 2.5. Let $(X, \mathcal{A})$ and $(Y, \mathcal{B})$ be two ample spaces. If a mapping $f: X \rightarrow Y$ satisfies $B \in \mathcal{B} \Rightarrow f^{-1}(B) \in \mathcal{A}$

then, $f$ is called a fuzzy variable from $(X, \mathcal{A})$ to $(Y, \mathcal{B})$.

Proposition 2.6. Let $(X, \mathcal{A})$ be an ample space and $\Pi$ is a possibility measure over $\mathcal{A}$. If $f$ is a fuzzy variable from $(X, \mathcal{A})$ to $(Y, \mathcal{B})$, then $\Pi\left(f^{-1}(B)\right), \forall B \in \mathcal{B}$ is a possibility measure over $\mathcal{B}$.

Proof. See [2], [4].

DeFinition 2.7. A cartesian closed category is a category $\mathcal{E}$ that satisfies the following;

(1) $\mathcal{E}$ is finitely complete,

(2) $\mathcal{E}$ has exponentiation. A topos is a cartesian closed category $\mathcal{E}$ that satisfies the following;

(3) $\mathcal{E}$ has a subobject classifier. 
EXAmple 2.8. ([1], [3]) Category Fuz of fuzzy sets is a cartesian closed category whose object is $\left(A, \alpha_{A}\right)$ where $A$ is an object and $\alpha_{A}: A \rightarrow I$ is a morphism with $I=(0,1]$ in Set and morphism from $\left(A, \alpha_{A}\right)$ to $\left(B, \alpha_{B}\right)$ is a function $f: A \rightarrow B$ in Set such that $\alpha_{A}(a) \leqq \alpha_{B} \circ f(a)$.

EXAMPLE 2.9. ([1], [3]) If $M_{2}$ is a monoid with two elements, then the category $M_{2}-\mathcal{S}$ et is a topos.

Consider $\left(M_{2}, \circ, e\right)$ where $M_{2}=\{e, a\}$ and $\circ$ is defined by $e \circ e=$ $e, e \circ a=a \circ e=a \circ a=a$. Then $M_{2}$ is a monoid with identity e, in which $a$ has no inverse. The set $L_{2}$ of left ideals of $M_{2}$ has three elements, that is, $M_{2}, \emptyset$, and $\{a\}$. Thus in $M_{2}-\mathcal{S} e t, \Omega=\left(L_{2}, \omega\right)$, where the action $\omega: M_{2} \times L_{2} \rightarrow L_{2}$ is defined by $\omega(m, B)=\{n \mid n \circ m \in B\}$.

Definition 2.10. Let $(X, \mathcal{A})$ and $(Y, \mathcal{B})$ be two ample spaces. Let the mapping $f: X \times \mathcal{B} \rightarrow[0,1]$ satisfy

(1) $\forall x \in X, f(x,-): \mathcal{B} \rightarrow[0,1]$ is a possibility measure.

(2) $\forall B \in \mathcal{B}, f(-, B): X \rightarrow[0,1]$ is a fuzzy variable, where ample field on $[0,1]$ is $P([0,1])$. Then $f$ is called a possibility mapping from $(X, \mathcal{A})$ to $(Y, \mathcal{B})$.

\section{Some properties of the category PosM}

Theorem 3.1. Terminal object exists in the Category PosM.

Proof. Let $1=(\{*\}, \mathcal{A})$ where $\mathcal{A}=\{\phi,\{*\}\}$. Then, for any $(Y, \mathcal{B})$, there exists a mapping $f: Y \times \mathcal{A} \rightarrow[0,1]$ defined by $f(y,\{*\})=1$ and $f(y, \phi)=0$ for all $y \in Y$. So, we get $f\left(y, \bigcup A_{i}\right)=\sup f\left(y, A_{i}\right)$. Thus $f(y,-)$ is a possibility measure. Also $f(-, A): Y \rightarrow[0,1]$ is a fuzzy variable. Since

$f(-, \phi)^{-1}(B)=Y$ if $0 \in B$

$f(-, \phi)^{-1}(B)=\phi$ otherwise. And

$f(-,\{*\})^{-1}(B)=Y$ if $1 \in B$

$f(-,\{*\})^{-1}(B)=\phi$ otherwise.

Therefore $f: Y \times \mathcal{A} \rightarrow[0,1]$ is a possibility mapping.

Theorem 3.2. Equalizer exists in the Category PosM.

Proof. $(X, \mathcal{A})$ and $(Y, \mathcal{B})$ are two ample spaces and $f, g:(X, \mathcal{A}) \rightarrow$ $(Y, \mathcal{B})$ are two possibility mappings. Let $E=\{x \in X \mid f(-, B)(x)=$ 
$g(-, B)(x) \forall B \in \mathcal{B}\}$ and $\mathcal{E}=\mathcal{A}$. And we construct a mapping $e$ : $E \times \mathcal{A} \rightarrow[0,1]$ defined by $e(a, A)=1$ if $a \in A$ $e(a, A)=0$ otherwise.

Then $e:(E, \mathcal{E}) \rightarrow(X, \mathcal{A})$ is a possibility mapping. Since $e(a,-)$ : $\mathcal{A} \rightarrow[0,1]$ is a possibility measure,

$e(a,-)(\phi)=0$,

$e(a,-)(X)=1$ and

$e(a,-)\left(\bigcup E_{i}\right)=\sup e(a,-)\left(E_{i}\right)$,

$e(-, A): E \rightarrow[0,1]$ is a fuzzy variable,

$e(-, A)^{-1}\left(E_{i}\right)=\{a \in E \mid e(a, A)=1\}$

$=\{a \in E \mid a \in A\}$ if $1 \in E_{i}$ and

$e(-, A)^{-1}\left(E_{j}\right)=\{a \in E \mid e(a, A)=0\}$

$=\left\{a \in E \mid a \in A^{c}\right\}$ if $0 \in E_{j}$.

Since

$f \circ e(x, B)=\vee(e(x,[a]) \wedge f(a, B))=f(a, B)$

by $(e(x,[a])=1$ or $(e(x,[a])=0$ and

$g \circ e(x, B)=\vee(e(x,[a]) \wedge g(a, B))=g(a, B)$

by $(e(x,[a])=1$ or $(e(x,[a])=0$,

we have $f \circ e=g \circ e$.

Theorem 3.3. Finite products exist in the Category PosM.

Proof. For any two ample spaces $(X, \mathcal{A})$ and $(Y, \mathcal{B})$, we construct an ample space $(Z, \mathcal{Z})$ where $Z=X \times Y$ and $\mathcal{E}=P(X) \times P(Y)$ with $p_{X}:(Z, \mathcal{Z}) \rightarrow(X, \mathcal{A})$ and $p_{Y}:(Z, \mathcal{Z}) \rightarrow(Y, \mathcal{B})$. Then $\left((Z, \mathcal{Z}), p_{X}, p_{Y}\right)$ is a product object of $(X, \mathcal{A})$ and $(Y, \mathcal{B})$.

We construct $p_{X}:(Z, \mathcal{Z}) \rightarrow(X, \mathcal{A})$ defined by

$p_{X}((x, y), A)=1$ if $x \in A$

$p_{X}((x, y), A)=0$ otherwise.

Then we have that $p_{X}:(Z, \mathcal{Z}) \rightarrow(X, \mathcal{A})$ is a possibility mapping. Since $p_{X}((a, b),-): \mathcal{A} \rightarrow[0,1]$ is a possibility measure,

$p_{X}((a, b),-)(\phi)=0$,

$p_{X}((a, b),-)(X)=1$ and

$p_{X}((a, b),-)\left(\bigcup A_{i}\right)=\sup p_{X}((a, b),-)\left(A_{i}\right)$,

also $p_{X}(-, A): Z \rightarrow[0,1]$ is a fuzzy variable,

$p_{X}(-, A)^{-1}\left(E_{i}\right)=\{(x, y) \mid x \in A\}$ if $1 \in E_{i}$ and

$p_{X}(-, A)^{-1}\left(E_{j}\right)=\{(x, y) \mid x \notin A\}$ if $0 \in E_{j}$.

For any possibility mappings $f:(K, \mathcal{K}) \rightarrow(X, \mathcal{A})$ and $g:(K, \mathcal{K}) \rightarrow$ $(Y, \mathcal{B})$, there exists a mapping $\langle f, g>:(K, \mathcal{K}) \rightarrow(Z, \mathcal{E})$ defined by 
$<f . g>(k,(A, B))=f(k, A)$ if $B$ is fixed

$<f . g>(k,(A, B))=g(k, B)$ if $A$ is fixed.

Then $\langle f, g\rangle$ is a possibility mapping. Since $x \notin A$ implies $p_{X}((x, y), A)=$ $0, x \in A$ implies $p_{X}((x, y), A)=1$ and $y$ is fixed we get

$\bigvee\left[<f, g>(k,[(x, y)]) \wedge p_{X}((x, y), A)\right]=f(f, A)$,

we have $p_{X} \circ<f, g>=f$.

Theorem 3.4. Exponentiation exists in the Category PosM.

Proof. For any two ample spaces $(X, \mathcal{A})$ and $(Y, \mathcal{B})$, we define $Y^{X}=$ $\{f \mid f:(X, \mathcal{A}) \rightarrow(Y, \mathcal{B})$ is a possibility mapping $\}$ and $\mathcal{D}=P\left(Y^{X}\right)$. Then $\left(Y^{X}, \mathcal{D}\right)$ is an object in the Category PosM. For any $Y^{X} \times X=$ $\{(f(-, Y), x) \mid f(-, Y)$ is a fuzzy variable $\}$ and $P\left(Y^{X}\right) \times P(X)=\mathcal{E}$, there exists a mapplng $e v: Y^{X} \times X \rightarrow Y$ defined by

$e v\left(\left(f\left(-, Y_{i}\right), x\right), Y_{j}\right)=0$ if $Y_{i} \cap Y_{j}=\phi$

$e v\left(\left(f\left(-, Y_{i}\right), x\right), Y_{j}\right)=1$ if $Y_{i} \cap Y_{j} \neq \phi$.

Then $e v:\left(Y^{X} \times X\right) \times \mathcal{B} \rightarrow[0,1]$ is a possibility measure, since

$e v\left(\left(f\left(-, Y_{i}\right), x\right), \phi\right)=0$

$e v\left(\left(f\left(-, Y_{i}\right), x\right), Y\right)=0$

$e v\left(\left(f\left(-, Y_{i}\right), x\right), \bigcup Y_{i}\right)=\sup e v\left(\left(f\left(-, Y_{i}\right), x\right), Y_{i}\right)$.

also $e v:\left(Y^{X} \times X\right) \times \mathcal{B} \rightarrow[0,1]$ is a fuzzy variable, since

$e v\left(-, Y_{j}\right)^{-1}(0)=\left(f\left(-, Y_{i}\right), a\right) \in \mathcal{E}$ with $Y_{i} \cap Y_{j}=\phi$

$e v\left(-, Y_{j}\right)^{-} 1(1)=\left(f\left(-, Y_{i}\right), a\right) \in \mathcal{E}$ with $Y_{i} \cap Y_{j} \neq \phi$.

So $e v:\left(Y^{X} \times X\right) \times \mathcal{B} \rightarrow[0,1]$ is a possibility mapping.

For any possibility mapping $g: Z \times X \rightarrow Y$ where $((Z, \mathcal{Z})$ is an ample space, there exists a mapping $\bar{g}:(Z, \mathcal{Z}) \rightarrow\left(Y^{X}, P\left(Y^{X}\right)\right)$ defined by $\bar{g}\left(z,\left(f\left(-, Y_{i}\right), x\right)=g\left((z, x), Y_{i}\right)\right.$. Then $\bar{g}:(Z, \mathcal{Z}) \rightarrow\left(Y^{X}, P\left(Y^{X}\right)\right)$ is a possibility mapping. So we have

$$
\begin{aligned}
& \bar{g} \times i d \circ \operatorname{ev}\left((z, x), Y_{i}\right) \\
& =\vee\left(\bar{g} \times i d\left((z, x),\left[\left(f\left(-, Y_{i}\right), x\right)\right]\right) \wedge \operatorname{ev}\left(\left(\left(f\left(-, Y_{i}\right), x\right), Y_{j}\right)\right)\right. \\
& =\bar{g} \times i d\left(z,\left[\left(f\left(-, Y_{i}\right), x\right)\right]\right) \\
& =\bar{g}\left(z,\left[\left(f\left(-, Y_{i}\right), x\right)\right]\right) \\
& =g\left((z, x), Y_{i}\right)
\end{aligned}
$$

Corollary 3.5. Category PosM is Cartesian closed.

Theorem 3.6. For each monic $f:(X, \mathcal{C}) \rightarrow(Y, \mathcal{D})$ where $f[X] \in \mathcal{D}$, a subobject classifier exists in the Category PosM. 
Proof. Let $2=\{0,1\}$ and $\mathcal{O}=\{\phi,\{0\},\{1\}, 2\}$. Then $\Omega=(2, \mathcal{O})$ is an ample space. We construct $T:(\{*\}, \mathcal{A}) \rightarrow(2, \mathcal{O})$ defined by $\top(*, \phi)=\top(*,\{0\})=0, \top(*,\{1\})=\top(*, 2)=1$. So, we get that $\top\left(*, \bigcup O_{i}\right)=\sup \top\left(*, O_{i}\right)$. Thus $\top(*,-)$ is a possibility measure. Also $\top(-, O):\{*\} \rightarrow[0,1]$ is a fuzzy variable since

$$
\begin{aligned}
& T^{-1}(-, 2)(0)=\phi \in \mathcal{A} \\
& T^{-1}(-,\{1\})(0)=\phi \in \mathcal{A} \\
& T^{-1}(-,\{0\})(0)=* \in \mathcal{A} \\
& T^{-1}(-, \phi)(0)=* \in \mathcal{A} \text { and } \\
& T^{-1}(-, 2)(1)=* \in \mathcal{A} \\
& T^{-1}(-,\{1\})(1)=* \in \mathcal{A} \\
& T^{-1}(-,\{0\})(1)=\phi \in \mathcal{A} \\
& T^{-1}(-, \phi)(1)=\phi \in \mathcal{A} .
\end{aligned}
$$

Hence $T$ is a possibility mapping. For any possibility mapping $f$ : $(X, \mathcal{C}) \rightarrow(Y, \mathcal{D})$ where $f[X] \in \mathcal{D}$, we construct a mapping $\chi_{f}:(Y, \mathcal{D}) \rightarrow$ $(2, \mathcal{O})$ defined by $\chi_{f}(y, \phi)=\chi_{f}(f(x),\{0\})=\chi_{f}(Y-f(x),\{1\})=0$ and $\chi_{f}(y, 2)=\chi_{f}(f(x),\{1\})=\chi_{f}(Y-f(x),\{0\})=1$. So we get

$\chi_{f}\left(Y, \bigcup A_{i}\right)=\sup \chi_{f}\left(Y, A_{i}\right)$.

Thus $\chi_{f}(Y,-)$ is a possibility measure. Also $\chi_{f}(-, O)$ is a fuzzy variable since

$$
\begin{aligned}
& \chi_{f}^{-1}(-, 2)(1)=Y \in \mathcal{D} \\
& \chi_{f}^{-1}(-,\{1\})(1)=f[X] \in \mathcal{D} \\
& \chi_{f}^{-1}(-,\{0\})(1)=Y-f[X] \in \mathcal{D} \\
& \chi_{f}^{-1}(-, \phi)(1)=\phi \in \mathcal{D} \text { and } \\
& \chi_{f}^{-1}(-, 2)(0)=\phi \in \mathcal{D} \\
& \chi_{f}^{-1}(-,\{1\})(0)=Y-f[X] \in \mathcal{D} \\
& \chi_{f}^{-1}(-,\{0\})(0)=f[X] \in \mathcal{D}
\end{aligned}
$$

Hence $\chi_{f}$ is a possibility mapping. Also we get $T \circ !=\chi_{f} \circ f$ since

$\top \circ !(x, \phi)=\chi_{f} \circ f(x, \phi)=0$

$\top \circ !(x,\{0\})=\chi_{f} \circ f(x,\{0\})=0$

$\top \circ !(x,\{1\})=\chi_{f} \circ f(x,\{1\})=1$

$\top \circ !(x, 2)=\chi_{f} \circ f(x, 2)=1$.

\section{References}

[1] R. Goldblatt, Topoi, North-Holland, 1984. 
[2] H.J. Janssen, G.De. Cooman, E.E. Kerre, Ample fields as a basis for possibilistic processes, Fuzzy Sets and System 120 (2001), 445-458.

[3] P.T. Johnstone, Topos Theory, Academic Press, N. Y. 1977.

[4] X.H. Yuan, H.X. Li, C. Zhang. The set-valued mapping based on ample fields, Computer and Mathematics with Applications 56 (2008), 1954-1965.

[5] P.Z. Wang, Fuzzy contactibility and fuzzy variables, Fuzzy Sets and System 8 (1982), 81-92.

Department of Data Information

Sangji University

Wonju 660, Korea

E-mail: iskim@sangji.ac.kr 